\section{ATHEROSCLEROSIS}

\section{$\mathrm{T}_{\text {reg }}$ cells promote plaque regression}

Regulatory $T\left(T_{\text {reg }}\right)$ cells have been shown to have anti-atherogenic functions, protecting against atherosclerosis development and progression. A new study now shows that $\mathrm{T}_{\text {reg }}$ cells also influence atherosclerosis regression by promoting the resolution of plaque inflammation through the suppression of macrophage and $T$ cell pro-inflammatory responses and the induction of a pro-resolving macrophage phenotype that facilitates tissue repair and plaque regression.

Kathryn Moore and colleagues show that $\mathrm{T}_{\text {reg }}$ cell numbers increase within regressing plaques in multiple mouse models of atherosclerosis regression. Single-cell RNA sequencing analyses revealed that the $T_{\text {reg }}$ cell profile changes during atherosclerotic plaque regression induced by lipid lowering, indicating that $T_{\text {reg }}$ cells from regressing plaques derive from peripheral differentiation of naive $T$ cells, whereas $\mathrm{T}_{\text {reg }}$ cells from progressing plaques derive from the thymus, and have increased activation and metabolic activity. The researchers also demonstrate that $T_{\text {reg }}$ cells are required for atherosclerosis regression. Antibody-mediated depletion of $\mathrm{T}_{\text {reg }}$ cells in mice blocked the atherosclerosis regression achieved with aggressive lipid-lowering therapy. Atherosclerotic plaques from mice depleted of $\mathrm{T}_{\text {reg }}$ cells had an inflammatory phenotype similar to that of progressing plaques and did not show hallmarks of inflammation resolution. The findings indicated that $\mathrm{T}_{\text {reg }}$ cells were required for pro-resolving processes during atherosclerosis regression, including decreased T helper 1 cell response, increased macrophage emigration, polarization of macrophages to an M2-like tissue-reparative phenotype, clearance of dying cells and stimulation of the production of and response to lipid mediators of inflammation resolution.

Together, these findings suggest that therapeutic expansion of $\mathrm{T}_{\text {reg }}$ cells, combined with lipid-lowering therapy, might be a promising approach to promote inflammation resolution and atherosclerosis regression.

Irene Fernández-Ruiz

ORIGINAL ARTICLE Sharma, M. et al. Regulatory T cells license macrophage pro-resolving functions during atherosclerosis regression. Circ. Res. https://doi.org/10.1161/ CIRCRESAHA.119.316461 (2020) RELATED ARTICLES Saigusa, R., Winkels, H. \& Ley, K. T cell subsets and functions in atherosclerosis. Nat. Rev. Cardiol. https://doi.org/10.1038/s41569-020-0352-5 (2020) | Bäck, M. et al. Inflammation and its resolution in atherosclerosis: mediators and therapeutic opportunities. Nat. Rev. Cardiol. 16, 389-406 (2019)

\title{
Clopidogrel is a favourable alternative to ticagrelor in older patients with NSTE-ACS
}

In patients aged $\geq 70$ years with non-STelevation acute coronary syndrome (NSTE-ACS), clopidogrel is associated with fewer bleeding events compared with ticagrelor but similar net clinical benefits (in terms of all-cause death, myocardial infarction, stroke and bleeding). This finding indicates that clopidogrel can be used as a first choice therapy for older patients who are at increased risk of bleeding.

Current guidelines recommend the use of ticagrelor or prasugrel over clopidogrel to prevent recurrent thrombotic events in patients with NSTE-ACS. However, bleeding risk increases with age, and ticagrelor has been associated with more frequent bleeding events than clopidogrel among older patients. To confirm these findings, investigators of the POPular AGE randomized trial sought to assess the bleeding risks of antiplatelet therapy in older patients with NSTE-ACS.

In total, 1,002 patients aged $\geq 70$ years with NSTE-ACS were randomly assigned to receive clopidogrel $(n=500)$ or either ticagrelor or prasugrel $(n=502$; referred to as ticagrelor group because the majority of the patients received ticagrelor). At 12 months, the primary bleeding outcome (composite of PLATO major or minor bleeding) occurred less frequently in the clopidogrel group than in the ticagrelor group $(18 \%$ versus $24 \%$, HR $0.71,95 \%$ CI $0.54-0.94$, $P=0.02$ ). However, the net clinical benefit outcome was similar between the two groups (28\% versus $32 \%$ ).

In summary, clopidogrel is associated with reduced bleeding risks compared with ticagrelor in patients aged $\geq 70$ years with NSTE-ACS. "Personalized antiplatelet therapy by means of platelet function testing or genotyping might further optimize antiplatelet therapy in older patients," comment the investigators.

Karina Huynh

ORIGINAL ARTICLE Gimbel, M. et al. Clopidogrel versus ticagrelor or prasugrel in patients aged 70 years or older with non-ST-elevation acute coronary syndrome (POPular AGE): the randomised, open-label, non-inferiority trial. Lancet 395 , 1374-1381 (2020)

\section{RISK FACTORS}

\section{Childhood adversity is associated with cardiovascular disease in later life}

An adverse childhood family environment (CFE) is associated with an increased rate of cardiovascular disease and death in middle age, according to a longitudinal cohort study.

A total of 3,646 participants in the CARDIA study who were recruited in 1985-1986 in the USA completed the CFE questionnaire in 2000-2001. The questionnaire assessed seven aspects of the family environment: parental support, verbal abuse, physical affection, physical abuse, presence of an alcohol or drug abuser, household management and parental knowledge of the child's activities. Researchers grouped participants into high, moderate and low relative CFE adversity scores.

At baseline, the mean age of participants was $25.1 \pm 3.6$ years, $47 \%$ were black and $56 \%$ were female. During follow-up (median 30.9 years), 198 participants developed cardiovascular disease. In fully adjusted models, the risk of developing cardiovascular disease (primarily coronary artery disease) was higher in participants who reported high CFE adversity (HR 1.40, 95\% Cl 0.98-2.11) or moderate CFE adversity (HR 1.25, 95\% Cl 0.89-1.75), relative to those who reported low CFE adversity. Similarly, the risk of all-cause death was higher in participants with high (HR 1.68, 95\% Cl 1.17-2.41) or moderate (HR 1.55, 95\% Cl 1.11-2.17) CFE adversity, relative to those with low CFE adversity.

The researchers speculate that children who are exposed to adverse emotional or traumatic experiences are more likely to develop behavioural risk factors for cardiovascular disease, such as smoking, anxiety, depression and a sedentary lifestyle. However, in an accompanying editorial, Donald Barr cautions that a child who experiences adversity is not irreversibly predestined to develop early-onset cardiovascular disease and should receive the appropriate support to avoid these outcomes.

Gregory B. Lim

ORIGINAL ARTICLE Pierce, J. B. et al. Association of childhood psychosocial environment with 30-year cardiovascular disease incidence and mortality in middle age. J. Am. Heart Assoc. 9, e015326 (2020) 